\title{
Clinical correlation of calpain-1 and glypican-3 expression with gallbladder carcinoma
}

\author{
WEIQING LUO ${ }^{1,2^{*}}$, ZHIGANG REN $^{1 *}$, SHENG GAO ${ }^{1}$, HAILONG JIN ${ }^{3}$, \\ GEER ZHANG $^{3}$, LIN ZHOU $^{1}$ and SHUSEN ZHENG ${ }^{1}$ \\ ${ }^{1}$ Key Laboratory of Combined Multi-Organ Transplantation, Ministry of Public Health, \\ Department of Hepatobiliary and Pancreatic Surgery, The First Affiliated Hospital, \\ School of Medicine, Zhejiang University, Hangzhou, Zhejiang 310003; \\ ${ }^{2}$ Department of General Surgery, The People's Hospital of Deqing County, Huzhou, Zhejiang 313200; \\ ${ }^{3}$ Department of Gastrointestinal Surgery, The First Affiliated Hospital, School of Medicine, \\ Zhejiang University, Hangzhou, Zhejiang 310003, P.R. China
}

Received December 3, 2014; Accepted November 27, 2015

DOI: $10.3892 / \mathrm{ol} .2016 .4079$

\begin{abstract}
Gallbladder carcinoma (GBC) possesses a poor prognosis, which is primarily attributed to the lack of early and timely surgical intervention. Calpain-1 and glypican-3 have been implicated in the progression of various types of cancer. The present study aimed to detect the expression of calpain-1 and glypican-3 in GBC, and analyzed whether the expression levels of these proteins correlated with any clinicopathological variables. A total of 100 patients with GBC and 30 patients with cholecystitis who accepted surgical treatment were enrolled in the present study. Pathological and clinical data were obtained from all patients. The expression of calpain-1 and glypican-3 was detected in paraffin-embedded tissues by immunohistochemistry. Calpain-1 expression was manually assessed with an immunohistochemical H-score with a slight modification. Glypican-3 expression was assessed as negative and positive. The correlations between protein expression and clinicopathological characteristics, and the associations between the proteins were analyzed. All patients exhibited positive expression of calpain-1. Notably, the high expression rate of calpain-1 was significantly increased in patients with GBC, compared with patients with cholecystitis (32.0 vs. 6.7\%; $\left.\chi^{2}=7.668 ; \mathrm{P}=0.006\right)$, suggesting that calpain-1 expression may
\end{abstract}

Correspondence to: Professor Shusen Zheng, Key Laboratory of Combined Multi-Organ Transplantation, Ministry of Public Health, Department of Hepatobiliary and Pancreatic Surgery, The First Affiliated Hospital, School of Medicine, Zhejiang University, 79 Qingchun Road, Hangzhou, Zhejiang 310003, P.R. China E-mail: shusenzheng@zju.edu.cn

*Contributed equally

Key words: gallbladder carcinoma, cholecystitis, calpain-1, glypican-3, immunohistochemistry be associated with progression from cholecystitis to GBC. In addition, the positive rate of glypican-3 expression was $53.0 \%$ in patients with $\mathrm{GBC}$ and $63.3 \%$ in patients with cholecystitis, with no significant difference $\left(\chi^{2}=0.997 ; \mathrm{P}=0.318\right)$. Furthermore, the expression of calpain-1 and glypican-3 had no significant correlation with gender, age, degree of tumor differentiation and tumor-node-metastasis classification in patients with GBC. Notably, the expression of calpain-1 and glypican-3 displayed a significant positive correlation in patients with GBC $(\mathrm{r}=0.517 ; \mathrm{P}<0.01)$, but a significantly negative correlation $(\mathrm{r}=-0.856 ; \mathrm{P}<0.01)$ in patients with cholecystitis. In conclusion, calpain-1 expression may be associated with progression from cholecystitis to GBC. Combined detection of calpain-1 and glypican-3 may be beneficial for prognosis assessment of GBC.

\section{Introduction}

Gallbladder carcinoma (GBC) is the most commonly observed malignancy of the biliary tract, representing $80-95 \%$ of all the cases of biliary tract cancer worldwide, and is the sixth most frequent malignant neoplasm of the digestive tract (1). In 2012, 76,844 patients were diagnosed with GBC, and $\sim 60,334$ patients succumbed to disease (2). Currently, the overall mean survival time for patients exhibiting $\mathrm{GBC}$ is 6 months, and the 5-year survival rate is 5\% (3). A positive clinical outcome may depend on early and timely surgical resection of GBC (4). However, $>90 \%$ of patients do not undergo surgical resection due to the advanced stage of their tumors at the time of diagnosis. Invasion of adjacent organs or distant metastases is observed in patients with advanced GBC, and almost $50 \%$ of them exhibit lymph node metastasis $(5,6)$. Thus, it is essential to identify novel prognostic biomarkers and therapeutic targets for the treatment of GBC.

The mammalian calpain protease family comprises intracellular $\mathrm{Ca}^{2+}$-regulated cysteine proteases that mediate regulatory cleavage of specific substrates (7). Calpains are involved in various physiological functions, including cell differentiation, transcriptional regulation, cytokine processing, 
cell cycle, signal transduction, migration, apoptosis and protein renewal during growth and tissue regeneration $(7,8)$. In addition, the calpain family, including calpain-1, has been observed to be involved in the progression of cancer $(8,9)$. The expression of calpain-1 has been reported to be associated with relapse-free survival in patients with breast cancer treated with trastuzumab following adjuvant chemotherapy (8), and was also correlated with increased malignancy in renal cell carcinoma (10).

Glypican-3 is a cell surface protein that attaches to the cell membrane via a glycosylphosphatidylinositol (GPI) anchor (11). Glypican-3 is able to combine with Wnt molecules to form a complex, thereby promoting cancer growth via stimulation of canonical Wnt signaling (12). It has been reported that glypican-3 is able to regulate developmental growth by interacting with the Hedgehog signaling pathway (13). Previous studies have revealed that mutated glypican-3 lacking its GPI anchor domain is able to block Wnt signaling and inhibit the growth of Wnt-dependent tumors $(14,15)$. Additional reports have demonstrated that glypican-3 expression is involved in various human malignancies, including hepatocellular carcinoma (HCC) (16), melanoma (17), ovarian clear cell carcinoma (18), yolk sac tumor (YST) (19), neuroblastoma, hepatoblastoma and Wilms' tumor, among others $(20,21)$.

However, to the best of our knowledge, the expression of calpain-1 and glypican-3 in GBC has not been investigated thus far. In the present study, the expression of calpain-1 and glypican-3 was detected in 100 patients with GBC and 30 patients with cholecystitis by immunohistochemistry, and the correlations between calpain-1 and glypican-3 expression and certain clinicopathological characteristics of the patients were analyzed.

\section{Materials and methods}

Clinical samples. The present study was performed according to REporting recommendations for tumor MARKer prognostic studies (REMARK) criteria (22). A total of 100 patients with GBC and 30 patients with cholecystitis who accepted surgical treatment between January 2007 and December 2011 in The First Affiliated Hospital, School of Medicine, Zhejiang University (Hangzhou, China) were enrolled in the study. Written informed consent was obtained from the patients prior to commencement of the study. The present study was approved by the Ethics Review Committee of The First Affiliated Hospital, School of Medicine, Zhejiang University (reference number 2014-332). The inclusion criteria of the patients with GBC were set as follows: i) The postoperative pathological diagnosis was GBC; ii) no radiotherapy or chemotherapy had been administered prior to surgery; iii) no comorbid diseases were present; and iv) complete pathological and clinical information was available, including age, gender, degree of tumor differentiation, tumor-node-metastasis (TNM) classification (23) and presence of distant metastases. The detailed clinicopathological variables of the patient cohort are presented in Table I.

Immunohistochemical staining. Paraffin-embedded GBC and cholecystitis tissues were sectioned with a thickness of $4 \mu \mathrm{m}$, and deparaffinized using xylene (Sangon Biotech
Co., Ltd., Shanghai, China). The slides were immersed into various concentrations of alcohol (100\%, 95\%, 75\% and 50\%; Sangon Biotech Co., Ltd.) diluted with double distilled $\mathrm{H}_{2} \mathrm{O}$ for rehydration, and subsequently treated with $3 \% \mathrm{H}_{2} \mathrm{O}_{2}$ (product code, M82228702; Sinopharm Chemical Reagent Co., Ltd., Shanghai, China) to block endogenous peroxidase activity. For antigen retrieval, the slides were immersed in boiling $\left(95-100^{\circ} \mathrm{C}\right.$ ) citrate buffer (pH 6.0; Sangon Biotech Co., Ltd.) for $20 \mathrm{~min}$. Upon washing with phosphate-buffered saline (PBS; Sangon Biotech Co., Ltd.), the slides were immersed into blocking solution (3\% bovine serum albumin; Hangzhou Sijiqing Bioengineering Material Co., Ltd., Hangzhou, China) at room temperature for $30 \mathrm{~min}$. Next, the slides were incubated overnight at $4^{\circ} \mathrm{C}$ with primary monoclonal mouse anti-rabbit calpain-1 (cat. no. ab3589; 1:1,000) and polyclonal rabbit anti-mouse glypican-3 (cat.no. ab66596; 1:1,000; Abcam, Cambridge, MA, USA) antibodies diluted in blocking serum. Following rinsing with PBS three times at room temperature, a horseradish peroxidase (HRP) polymer (SuperPicture ${ }^{\mathrm{TM}}$ Polymer Detection Kit, HRP, broad spectrum; Thermo Fisher Scientific, Inc., Waltham, MA, USA) conjugated to undiluted anti-rabbit (cat no. PV-6001) or anti-mouse (cat no. PV-6002) secondary antibodies (Zhongshan Golden Bridge Biotechnology Co., Ltd., Beijing, China) were added to the slides for $10 \mathrm{~min}$, and 3,3'-diaminobenzidine chromogen was then added for $5 \mathrm{~min}$. Following each incubation step, the slides were washed in PBS for 5 min. Mayer's Hematoxylin Solution (Sigma-Aldrich, St. Louis, MO, USA) was utilized for counterstaining. Subsequently, the slides were dehydrated, air-dried and mounted with neutral resins (product code, ZLI-9555; Zhongshan Golden Bridge Biotechnology Co., Ltd.).

Assessment of staining was conducted by scanning the slides with an inverted microscope (BX41; Olympus Corporation, Tokyo, Japan) at magnification, x200. The expression of calpain-1 in tumor and cholecystitis cells was manually assessed by immunohistochemical $\mathrm{H}$-score, with a slight modification to the method previously described (8). Staining intensity was assessed as negative (0), weak (1), medium (2) or strong (3) over each stained area. The stained area score was assessed as $<50 \%$ (1) or $\geq 50 \%$ (2). H-scores were calculated by multiplying the stained area score by the staining intensity score (H-score range, 0-6). Calpain-1 H-score was dichotomized into low and high immunoreactivity groups using X-Tile Software (http://medicine.yale.edu/lab/rimm/research/software. aspx), and correlated with clinicopathological criteria. The expression of glypican-3 in tumor and cholecystitis cells was manually assessed as negative $\left({ }^{-}\right)$or positive $\left(^{+}\right)$. A total of $50 \%$ of the slides were examined by a second independent assessor who was blinded to the scores and clinicopathological criteria, and good concordance existed between the two scorers (single measure intraclass correlations, $>0.8$ ). An average $\mathrm{H}$-score was generated by calculating the mean of 10 random high-power fields. Average scores were utilized for analysis due to the relatively small sample size.

Statistical analysis. The data distribution was assessed using the Kolmogorov-Smirnov test for goodness of fit. The correlation between protein expression and clinicopathological characteristics was analyzed with Pearson's $\chi^{2}$ test of association or Fisher's exact test in a $2 \times 2$ table. Spearman's rank-order correlations 
Table I. Clinicopathological variables of the patient cohort.

\begin{tabular}{lcc}
\hline Clinicopathological variables & Gallbladder carcinoma $(\mathrm{n}=100)$ & Cholecystitis $(\mathrm{n}=30)$ \\
\hline Age, years & $63.57 \pm 0.99^{\mathrm{a}}$ & $54.13 \pm 1.99^{\mathrm{b}}$ \\
Tumor size $\mathrm{mm}^{3}$ & $29.55 \pm 7.20^{\mathrm{c}}$ & \\
Gender, $\mathrm{n}(\%)$ & $57(57)$ \\
Female & $43(43)$ \\
Male & \\
Differentiation degree, $\mathrm{n}(\%)$ & $73(73)$ \\
Poor and moderate & $27(27)$ \\
Well & \\
Tumor-node-metastasis classification, $\mathrm{n}(\%)$ & $17(17)$ \\
I+II & $83(83)$ \\
III+IV & \\
Distant metastases, $\mathrm{n}(\%)$ & $72(72)$ \\
Positive & $28(28)$ \\
Negative & \\
\hline
\end{tabular}

Continuous data are presented as the mean \pm standard error. ${ }^{\text {RRange, }} 40-86$ years ${ }^{\mathrm{b}}$ Range, $34-74$ years. ${ }^{\mathrm{c}} \mathrm{Range}, 0.22-625.00 \mathrm{~mm}^{3}$. Tumor-node-metastasis classification as referred to in the American Joint Committee on Cancer staging classification of gallbladder carcinoma, 7 th edition (23).
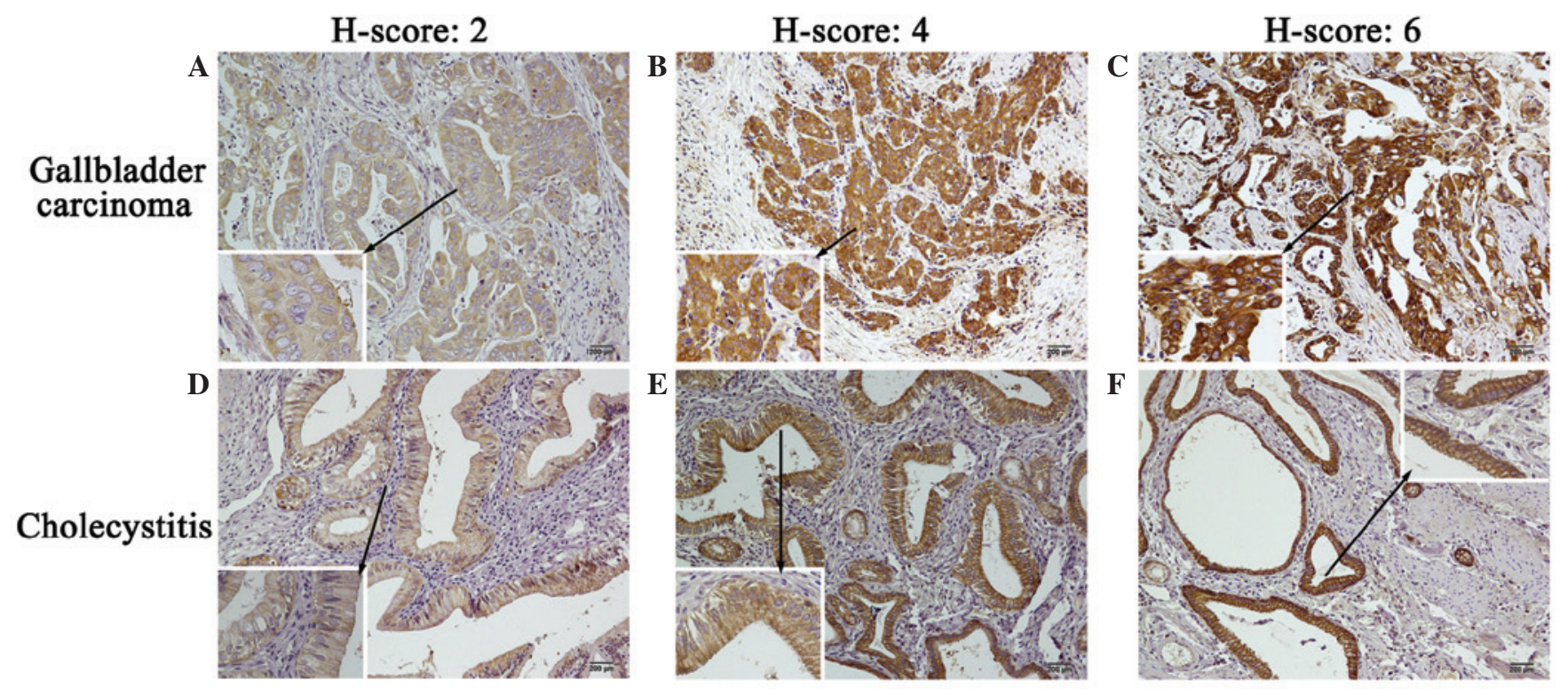

Figure 1. Immunohistochemical patterns of calpain-1 expression at magnification, $\mathrm{x} 200$, with inset panel at magnification, $\mathrm{x} 400$ and scale bar representing $200 \mu \mathrm{m}$. (A) Weak, (B) medium and (C) strong staining of calpain-1 in GBC tissues. (D) Weak, (E) medium and (F) strong staining of calpain-1 in cholecystitis tissues. GBC, gallbladder carcinoma.

were performed to investigate the associations between various proteins. $\mathrm{P}<0.05$ was considered to indicate a statistically significant difference. Statistical analyses were performed using SPSS software version 17.0 (SPSS Inc., Chicago, IL) for Windows (Microsoft Corporation, Redmond, WA, USA).

\section{Results}

Positive immunohistochemistry results are observed for calpain-1 and glypican-3. Tissue expression of calpain-1 and glypican-3 was investigated in patients with GBC and patients with cholecystitis. Representative staining patterns of calpain-1 expression in GBC and cholecystitis tissues are presented in Fig. 1. Cytomembrane and cytoplasmic staining was observed for calpain-1, with certain granularity and heterogeneity between adjacent tumor cells and adjacent cholecystitis cells, varying from weak to strong staining. According to the computational formula of $\mathrm{H}$-scores, the different intensities of calpain-1 expression (ranging from weak to strong) corresponded to distinct $\mathrm{H}$-scores (ranging from 2 to 6 ) in the GBC 


\section{Glypican-3 positive}

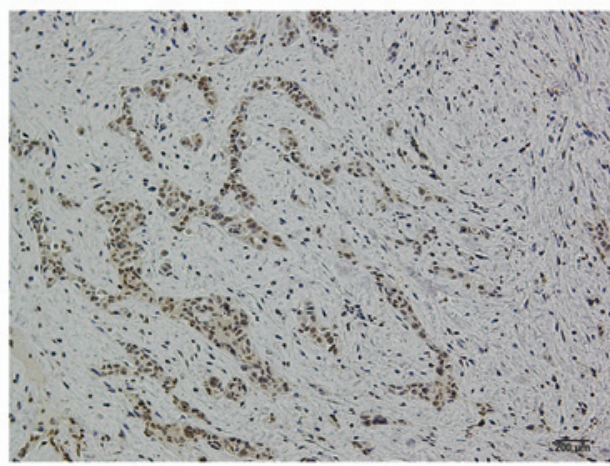

Gallbladder carcinoma

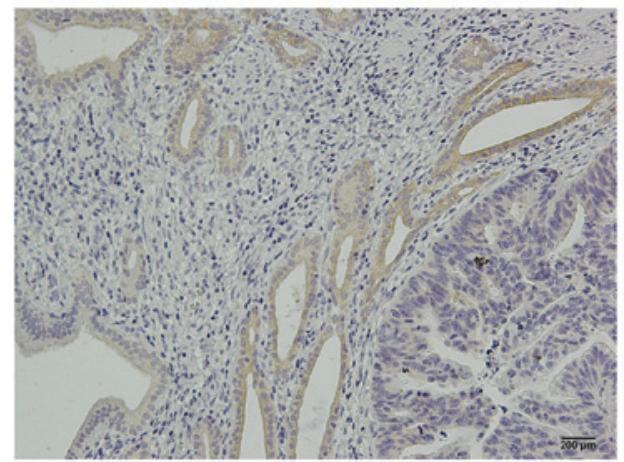

Cholecystitis

Figure 2. Immunohistochemical patterns of glypican-3 expression at magnification, x200 with scale bar representing $200 \mu \mathrm{m}$. Positive staining of glypican-3 in gallbladder carcinoma and cholecystitis tissues.

Table II. Expression distribution of calpain-1 and glypican-3 in patients with GBC and patients with cholecystitis.

\begin{tabular}{lccccc}
\hline & \multicolumn{2}{c}{ Calpain-1 $^{\text {a }}$} & & \multicolumn{2}{c}{ Glypican-3 } \\
\cline { 2 - 3 } \cline { 5 - 6 } Expression distribution & Low, $\mathrm{n}(\%)$ & High, $\mathrm{n}(\%)$ & & Negative, $\mathrm{n}(\%)$ & Positive, $\mathrm{n}(\%)$ \\
\hline GBC $(\mathrm{n}=100)$ & $68(68.0)$ & $32(32.0)$ & & $47(47.0)$ & $53(53.0)$ \\
Cholecystitis $(\mathrm{n}=30)$ & $28(93.3)$ & $2(6.7)$ & & $11(36.7)$ & $19(63.3)$ \\
$\chi^{2} /$ P-value $^{\mathrm{b}}$ & \multicolumn{2}{c}{$7.668 / 0.006$} & & $0.997 / 0.318$ \\
\hline
\end{tabular}

${ }^{a}$ Calpain-1 expression was manually assessed using a slightly modified immunohistochemical H-score, as follows: 0, negative expression; $1-3$, low expression; and 4-6, high expression. ${ }^{b}$ Fisher's exact (2x2) test was used to determine significance. GBC, gallbladder carcinoma.

(Fig. 1A-C) and cholecystitis (Fig. 1D-F) tissues. In 100 GBC tissue samples, calpain-1 exhibited a median $\mathrm{H}$-score of 2.73 and a standard error of 0.18 , while the median $\mathrm{H}$-score observed for calpain-1 in 30 cholecystitis tissues was $2.47 \pm 0.28$.

Furthermore, as demonstrated in Fig. 2, glypican-3 expression presented as cytomembrane and cytoplasmic staining in positively stained GBC and cholecystitis cells, whereas negative staining was exhibited by a number of cells. The positive expression rate of glypican-3 was $53.0 \%$ (53/100) in GBC tissues and 63.3\% (19/30) in cholecystitis tissues.

Differential distribution of calpain-1 and glypican-3 expression is observed in patients with GBC and patients with cholecystitis. H-score cut-offs were as follows: 0 , negative expression; 1-3, low expression; and 4-6, high expression. All 100 patients with GBC and 30 patients with cholecystitis presented positive expression of calpain-1, thus exhibiting a $100.0 \%$ positive expression rate for this protein. Of the 100 patients with GBC, 32 exhibited high expression levels of calpain-1 and 68 exhibited low expression levels, resulting in a high expression rate of $32.0 \%$. Of the 30 patients with cholecystitis, 2 exhibited high expression levels of calpain-1 and 28 exhibited low expression levels. Therefore the high expression rate for this protein was $6.7 \%$. Pearson's $\chi^{2}$ test demonstrated that the high expression rate of calpain-1 was significantly increased in patients with GBC, compared with patients with cholecystitis $\left(\chi^{2}=7.668 ; \mathrm{P}=0.006\right.$; Table II $)$.
Glypican-3 expression was manually assessed as negative (-) or positive $\left(^{+}\right)$. Of the 100 patients with GBC, 53 presented positive glypican-3 expression and 47 demonstrated negative expression; therefore the positive expression rate was $53.0 \%$. Of the 30 patients with cholecystitis, 19 demonstrated positive glypican-3 expression and 11 exhibited negative expression, therefore the positive expression rate was $63.3 \%$. Pearson's $\chi^{2}$ test indicated no significant difference in the positive expression rate of glypican-3 between patients with GBC and patients with cholecystitis $\left(\chi^{2}=0.997 ; \mathrm{P}=0.318\right.$; Table II).

No significant associations exist between calpain-1 and glypican-3 expression and various clinicopathological variables in patients with GBC. The correlation of calpain-1 expression with clinicopathological variables in patients with GBC was analyzed using Fisher's exact $(2 \times 2)$ test, as shown in Table III. With regard to gender, 21 female patients presented high expression levels of calpain-1 and 36 demonstrated low expression, while 11 male patients exhibited high expression levels of calpain-1 and 32 demonstrated low expression. Fisher's exact $(2 \times 2)$ test indicated no significant difference $\left(\chi^{2}=1.428 ; \mathrm{P}=0.232\right)$. With regard to age, 20 patients presented high expression levels of calpain-1 and 41 demonstrated low expression levels in the $\geq 60$ years group, while 12 patients exhibited high expression levels of calpain-1 and 27 demonstrated low expression levels in the $<60$ years group. Fisher's exact test indicated no significant 
Table III. Correlations between calpain-1 and glypican-3 expression and clinicopathologic variables in 100 patients with GBC.

\begin{tabular}{|c|c|c|c|c|c|c|}
\hline \multirow[b]{2}{*}{ GBC $(n=100)$} & \multicolumn{3}{|c|}{ Calpain-1 expression } & \multicolumn{3}{|c|}{ Glypican-3 expression } \\
\hline & Low & High & $\chi^{2} / \mathrm{P}-$ value $^{\mathrm{a}}$ & Negative & Positive & $\chi^{2} / \mathrm{P}$-value \\
\hline Gender & & & $1.428 / 0.232$ & & & $1.903 / 0.168$ \\
\hline Male & 32 & 11 & & 21 & 22 & \\
\hline Female & 36 & 21 & & 26 & 31 & \\
\hline Age, years & & & $0.045 / 0.833$ & & & $0.018 / 0.892$ \\
\hline$<60$ & 27 & 12 & & 18 & 21 & \\
\hline$\geq 60$ & 41 & 20 & & 29 & 32 & \\
\hline Degree of differentiation & & & $0.431 / 0.511$ & & & $1.474 / 0.225$ \\
\hline Poor and moderate & 51 & 22 & & 37 & 36 & \\
\hline Well & 17 & 10 & & 10 & 17 & \\
\hline Tumor-node-metastasis classification & & & $2.134 / 0.144$ & & & $1.127 / 0.288$ \\
\hline $\mathrm{I}+\mathrm{II}$ & 9 & 8 & & 6 & 11 & \\
\hline III+IV & 59 & 24 & & 41 & 42 & \\
\hline
\end{tabular}

${ }^{a}$ Fisher's exact $(2 \times 2)$ test was used to determine significance. GBC, gallbladder carcinoma.

Table IV. Correlation between calpain-1 and glypican-3 expression in 100 patients with GBC and 30 patients with cholecystitis.

\begin{tabular}{|c|c|c|c|c|}
\hline Patients & Calpain-1 & Glypican-3 $\left(^{+}\right), \mathrm{n}$ & Glypican-3 $\left(^{-}\right), \mathrm{n}$ & $\mathrm{r} / \mathrm{P}-$ value $^{\mathrm{a}}$ \\
\hline \multirow[t]{2}{*}{$\operatorname{GBC}(n=100)$} & High & 29 & 3 & $0.517 /<0.01$ \\
\hline & Low & 24 & 44 & \\
\hline \multirow[t]{2}{*}{ Cholecystitis $(n=30)$} & High & 1 & 18 & $-0.856 /<0.01$ \\
\hline & Low & 10 & 1 & \\
\hline
\end{tabular}

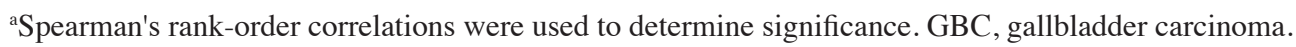

difference $\left(\chi^{2}=0.045 ; \mathrm{P}=0.833\right)$. With regard to the degree of tumor differentiation, 22 patients presented high expression levels of calpain-1 and 51 demonstrated low expression levels in the poor and moderate differentiation group, while 10 patients exhibited high expression and 17 low expression in the well-differentiated group. Fisher's exact test indicated no significant difference $\left(\chi^{2}=0.431 ; \mathrm{P}=0.511\right)$. With regard to TNM classification, 8 patients presented high expression levels of calpain-1 and 9 demonstrated low expression for stages I+II, while 24 patients exhibited high expression levels of calpain-1 and 59 demonstrated low expression for stages III+IV. Fisher's exact test indicated no significant difference $\left(\chi^{2}=2.134 ; \mathrm{P}=0.144\right)$. The results of the present study suggested that the expression of calpain-1 had no significant correlation with gender, age, tumor differentiation degree and TNM classification in patients with GBC.

The correlation of glypican-3 expression with clinicopathological variables in the patients with GBC presented a similar pattern to that mentioned above for calpain-1 expression (Table III). With regard to gender, 31 female patients presented positive glypican-3 expression and 26 negative, while 22 male patients demonstrated positive expression and 21 negative. Fisher's exact test indicated no significant difference $\left(\chi^{2}=1.903 ; P=0.168\right)$. With regard to age, 32 patients presented positive glypican-3 expression and 29 negative in the $\geq 60$ years group, while 21 patients demonstrated positive expression and 18 negative in the $<60$ years group. Fisher's exact test indicated no significant difference $\left(\chi^{2}=0.018\right.$; $\mathrm{P}=0.892)$. With regard to tumor differentiation, 36 patients presented positive glypican-3 expression and 37 negative in the poor and moderate differentiation group, while 17 patients were positive and 10 were negative in the well-differentiated group. Fisher's exact test indicated no significant difference $\left(\chi^{2}=1.474\right.$; $\mathrm{P}=0.225)$. With regard to TNM classification, 11 patients presented positive glypican-3 expression and 6 negative for stages I+II, while 42 patients were positive and 41 were negative for stages III+IV. Fisher's exact test indicated no significant difference $\left(\chi^{2}=1.127 ; \mathrm{P}=0.288\right)$. The results of the present study suggested that the expression of glypican-3 had no significant correlation with gender, age, tumor differentiation degree and TNM classification in patients with GBC.

Varying correlations are observed between calpain-1 and glypican-3 expression in patients with GBC and patients with cholecystitis. As presented in Table IV, in the GBC group, 29 patients presented high expression of calpain-1 and positive expression of glypican-3, 44 patients demonstrated low expression of calpain-1 and negative expression of glypican-3, 
3 patients demonstrated independent high expression of calpain-1 and 24 patients demonstrated independent positive expression of glypican-3. Spearman's rank-order correlations indicated that the expression of calpain-1 and glypican-3 in patients with GBC presented a significantly positive correlation $(\mathrm{r}=0.517 ; \mathrm{P}<0.01)$.

In the cholecystitis group, 1 patient presented high expression of calpain-1 and positive expression of glypican-3, 1 patient demonstrated low expression of calpain-1 and negative expression of glypican-3, 18 patients demonstrated independent high expression of calpain-1 and 10 patients demonstrated independent positive expression of glypican-3. Spearman's correlation analysis indicated that the expression of calpain-1 and glypican-3 in patients with cholecystitis presented a significantly negative correlation $(\mathrm{r}=-0.856 ; \mathrm{P}<0.01)$.

\section{Discussion}

GBC always results in advanced disease with invasion of adjacent organs or distant metastases at the time of presentation, primarily attributed to its relatively low incidence and unclear symptomatology, thereby leading to poor prognosis and reduced survival rates (24). Previous reports have demonstrated that several risk factors including age, parity, gender, obesity and ethnicity may be associated with $\operatorname{GBC}(24,25)$. The initiation and development of GBC may be due to a wide range of etiologies, including infectious and environmental exposure to chemical carcinogens, mechanical obstruction via gallstones, autoimmune disease, polyps, adenomas and anatomical variations such as pancreaticobiliary malfunction $(24,25)$. Although significant progress has been made to identify potential prognostic biomarkers for GBC, this disease remains an uncommon and challenging malignancy with an overall poor prognosis (25).

Carbohydrate antigen 19-9 and carcinoembryonic antigen are the most commonly used clinical biomarkers in GBC (26). However, they are frequently increased in the advanced stages of the disease with a low specificity, and therefore, they are not generally used independently for GBC prognosis (25). Previous studies have demonstrated that calpain-1 may be involved in the progression of certain types of cancer, including breast cancer (8) and renal cell carcinoma (10). Furthermore, a number of reports have indicated that glypican-3 expression may be involved in various types of cancer, including HCC (16), melanoma (17), ovarian clear cell carcinoma (18) and others $(20,21)$. However, to the best of our knowledge, the expression of calpain-1 and glypican-3 in GBC has not been investigated to date.

In the present study, the expression of calpain-1 and glypican-3 was detected in 100 patients with GBC and 30 patients with cholecystitis by immunohistochemistry, and the correlations between calpain-1 and glypican-3 expression and the clinicopathological characteristics of the patients were analyzed. It was identified that all patients presented positive expression of calpain-1. Notably, the high expression rate of calpain-1 in patients with GBC was markedly increased compared with that observed in patients with cholecystitis. Furthermore, the expression of calpain-1 and glypican-3 had no significant correlation with gender, age, degree of tumor differentiation and TNM classification in patients with GBC. Notably, calpain-1 and glypican-3 expression presented a significantly positive correlation in patients with GBC, but a significantly negative correlation in patients with cholecystitis.

The results of the present study are interesting in the light of previous studies highlighting the role of calpain-1 in the progression of cancer $(8,27,28)$. Kulkarni et al (27) investigated the role of calpain-1 in trastuzumab-treated human epidermal growth factor receptor 2 (HER2) ${ }^{+}$breast cancer in vitro. The authors demonstrated that calpain-1 was activated following trastuzumab treatment, and subsequently cleaved HER2, thus disrupting signaling, while trastuzumab-resistant cells were dependent on calpain-1 activity for survival (27). Storr et al (8) reported that calpain-1 expression was associated with relapse-free survival, and proposed that calpain-1 expression may be a useful biomarker for the prediction of relapse-free survival in patients with breast cancer treated with adjuvant trastuzumab and chemotherapy. An additional study by Storr et al (28) demonstrated that the expression of calpain-1 and calpastatin were associated with various clinicopathological features, including tumor grade and estrogen receptor expression, which was verified in an independent cohort of patients. Furthermore, Storr et al (29) investigated the protein expression levels of calpastatin and calpain-1, -2 and -9 in surgically excised gastroesophageal adenocarcinomas derived from patients treated with neoadjuvant chemotherapy and in tumors that had not been previously exposed to chemotherapy, and identified that expression of the calpain system was associated with poor clinical outcomes. Therefore, the authors proposed that calpain-1, calpain-2 and calpastatin may be clinically relevant prognostic biomarkers in gastroesophageal adenocarcinoma (29). In addition, Storr et al (30) indicated that the expression of these proteins was significantly associated with carcinoma of the pancreas, bile duct and ampulla, and influenced the progression of disease.

The potential mechanisms by which the calpain family participates in cancer progression are associated with a number of interrelated signaling pathways (31). Integrin engagement is able to induce focal adhesion kinase (FAK) phosphorylation, resulting in extracellular signal-regulated kinase activation of calpain-1 to cleave FAK, which subsequently enhances cell motility (32). FAK, like phosphatidylinositol (3,4,5)-trisphosphate 3-phosphatase, may be dephosphorylated by phosphatase and tensin homolog, which is indicative of signaling pathway overlap (32). These interrelated signaling pathways synergistically contribute to the progression of various types of cancer $(31,33,34)$. In the present study, all patients with GBC presented positive expression of calpain-1, suggesting that calpain-1 expression may be associated with GBC. However, the expression of calpain-1 had no significant correlation with gender, age, degree of tumor differentiation and TNM classification in these patients, which suggested that calpain-1 may be a potentially useful biomarker for GBC prognosis. Notably, the results of the present study demonstrated that the high expression rate of calpain-1 in patients with GBC was markedly increased compared with that observed in patients with cholecystitis ( 32.0 vs. $6.7 \%$; $\mathrm{P}=0.006)$. Thus, it may be speculated that the expression of calpain-1 is associated with progression from cholecystitis to GBC.

Glypican-3 is a cell surface protein that is highly expressed in certain types of human cancer, including HCC and melanoma $(17,35)$. It is associated with cell proliferation and survival, possibly due to its interaction with insulin-like growth 
factor (IGF) 2 (11). Song et al (36) mated glypican-3 knockout mice with insulin receptor substrate 1 nullizygous mice, and demonstrated that glypican-3 regulated organism growth independently of IGF signaling. Notably, glypican-3 knockout mice exhibited changes in Wnt signaling (36). Glypican-3 is able to form a complex with Wnt molecules, thereby promoting cancer growth by stimulation of canonical Wnt signaling (12), and is also able to regulate developmental growth via interaction with the Hedgehog signaling pathway (13). In addition, glypican-3 is able to act as a negative regulator of Hedgehog signaling during mammalian development (13). A number of studies have indicated that mutated glypican-3 lacking the GPI anchor domain was able to block Wnt signaling and inhibit the growth of Wnt-dependent tumors $(14,15)$. Previous studies have reported that glypican-3 expression is associated with various types of cancer, including HCC (16), melanoma (17), ovarian clear cell carcinoma (18), YST (19), neuroblastoma, hepatoblastoma, Wilms' tumor and others $(20,21)$. The present study demonstrated that 53 patients with GBC presented positive expression of glypican-3 (53.0\%), which suggested that glypican-3 expression may be associated with GBC.

The combined detection of multiple molecular markers is able to enhance the specificity and sensitivity of tumor prognostic assessment, and has become a potentially effective method for the prediction of tumor prognosis $(37,38)$. The present study identified a significantly positive correlation between the expression of calpain- 1 and glypican-3 in patients with GBC, and a significantly negative correlation in patients with cholecystitis via Spearman's correlation analysis. Thus, it may be speculated that the combined detection of calpain-1 and glypican-3 may be beneficial for the prognostic assessment of GBC. Identification and validation of additional patient studies on various human populations at risk of developing GBC, which include clinicopathological variables and prognostic values, is required in order to provide further insight into calpain-1 and glypican-3 detection as a potential prognostic biomarker for GBC.

In conclusion, the present study demonstrated that calpain-1 expression was associated with GBC, and may be a useful potential biomarker for the assessment of prognosis in patients with GBC. Notably, the expression of calpain-1 may be associated with progression from cholecystitis to GBC. Furthermore, combined detection of calpain- 1 and glypican-3 may be beneficial for the assessment of prognosis in patients with GBC.

\section{Acknowledgements}

The present study was supported by the National Science and Technology Major Projects of the Chinese Ministry of Science and Technology (Beijing, China) (grant no. 2012ZX10002-017), the National Natural Science Foundation of China for Innovative Research Group of China (Beijing, China) (grant no. 81421062) and the Medical and Health Science and Techonology Project of Zhejiang Province (Hangzhou, China) (grant no. 2014KYA210).

\section{References}

1. Hundal R and Shaffer EA: Gallbladder cancer: Epidemiology and outcome. Clin Epidemiol 6: 99-109, 2014.
2. Ferlay J, Soerjomataram I, Dikshit R, Eser S, Mathers C, Rebelo M, Parkin DM, Forman D and Bray F: Cancer incidence and mortality worldwide: Sources, methods and major patterns in GLOBOCAN 2012. Int J Cancer 136: E359-E386, 2015.

3. Henson DE, Albores-Saavedra J and Corle D: Carcinoma of the gallbladder. Histologic types, stage of disease, grade, and survival rates. Cancer 70: 1493-1497, 1992.

4. Åndrén-Sandberg A and Deng Y: Aspects on gallbladder cancer in 2014. Curr Opin Gastroenterol 30: 326-331, 2014.

5. Sheth S, Bedford A and Chopra S: Primary gallbladder cancer: Recognition of risk factors and the role of prophylactic cholecystectomy. Am J Gastroenterol 95: 1402-1410, 2000.

6. Misra S, Chaturvedi A, Misra NC and Sharma ID: Carcinoma of the gallbladder. Lancet Oncol 4: 167-176, 2003.

7. Benyamin Y: The structural basis of calpain behavior. FEBS J 273: 3413-3414, 2006.

8. Storr SJ, Woolston CM, Barros FF, Green AR, Shehata M, Chan SY, Ellis IO and Martin SG: Calpain-1 expression is associated with relapse-free survival in breast cancer patients treated with trastuzumab following adjuvant chemotherapy. Int J Cancer 129: 1773-1780, 2011.

9. Huang Y and Wang KK: The calpain family and human disease. Trends Mol Med 7: 355-362, 2001.

10. Kamei M, Webb GC, Young IG and Campbell HD: SOLH, a human homologue of the Drosophila melanogaster small optic lobes gene is a member of the calpain and zinc-finger gene families and maps to human chromosome 16p13.3 near CATM (cataract with microphthalmia). Genomics 51: 197-206, 1998.

11. Ho M and Kim H: Glypican-3: A new target for cancer immunotherapy. Eur J Cancer 47: 333-338, 2011.

12. Capurro MI, Xiang YY, Lobe C and Filmus J: Glypican-3 promotes the growth of hepatocellular carcinoma by stimulating canonical Wnt signaling. Cancer Res 65: 6245-6254, 2005.

13. Capurro MI, Xu P, Shi W, Li F, Jia A and Filmus J: Glypican-3 inhibits Hedgehog signaling during development by competing with patched for Hedgehog binding. Dev Cell 14: 700-711, 2008.

14. Zittermann SI, Capurro MI, Shi W and Filmus J: Soluble glypican 3 inhibits the growth of hepatocellular carcinoma in vitro and in vivo. Int J Cancer 126: 1291-1301, 2010.

15. Feng M, Kim H, Phung Y and Ho M: Recombinant soluble glypican 3 protein inhibits the growth of hepatocellular carcinoma in vitro. Int J Cancer 128: 2246-2247, 2011.

16. Hsu HC, Cheng W and Lai PL: Cloning and expression of a developmentally regulated transcript MXR7 in hepatocellular carcinoma: Biological significance and temporospatial distribution. Cancer Res 57: 5179-5184, 1997.

17. Nakatsura T, Kageshita T, Ito S, Wakamatsu K, Monji M, Ikuta Y, Senju S, Ono T and Nishimura Y: Identification of glypican-3 as a novel tumor marker for melanoma. Clin Cancer Res 10: 6612-6621, 2004

18. Stadlmann S, Gueth U, Baumhoer D, Moch H, Terracciano L and Singer G: Glypican-3 expression in primary and recurrent ovarian carcinomas. Int J Gynecol Pathol 26: 341-344, 2007.

19. Zynger DL, Dimov ND, Luan C, Teh BT and Yang XJ: Glypican 3: A novel marker in testicular germ cell tumors. Am J Surg Pathol 30: 1570-1575, 2006.

20. Baumhoer D, Tornillo L, Stadlmann S, Roncalli M, Diamantis EK and Terracciano LM: Glypican 3 expression in human nonneoplastic, preneoplastic, and neoplastic tissues: A tissue microarray analysis of 4,387 tissue samples. Am J Clin Pathol 129: 899-906, 2008.

21. Saikali Z and Sinnett D: Expression of glypican 3 (GPC3) in embryonal tumors. Int J Cancer 89: 418-422, 2000.

22. McShane LM, Altman DG, Sauerbrei W, Taube SE, Gion M and Clark GM; Statistics Subcommittee of the NCI-EORTC Working Group on Cancer Diagnostics: REporting recommendations for tumor MARKer prognostic studies (REMARK). Nat Clin Pract Oncol 2: 416-422, 2005.

23. Edge SB, Byrd DR, Compton CC, et al (eds.): Gallbladder. In: AJCC Cancer Staging Manual. 7th edition. Springer, New York, NY, pp211-217, 2010.

24. Boutros C, Gary M, Baldwin K and Somasundar P: Gallbladder cancer: Past, present and an uncertain future. Surg Oncol 21: e183-e191, 2012.

25. Srivastava K, Srivastava A and Mittal B: Potential biomarkers in gallbladder cancer: Present status and future directions. Biomarkers 18: 1-9, 2013.

26. Wang YF, Feng FL, Zhao XH, Ye ZX, Zeng HP, Li Z, Jiang XQ and Peng $\mathrm{ZH}$ : Combined detection tumor markers for diagnosis and prognosis of gallbladder cancer. World J Gastroenterol 20: 4085-4092, 2014. 
27. Kulkarni S, Reddy KB, Esteva FJ, Moore HC, Budd GT and Tubbs RR: Calpain regulates sensitivity to trastuzumab and survival in HER2-positive breast cancer. Oncogene 29: $1339-1350,2010$

28. Storr SJ, Lee KW, Woolston CM, Safuan S, Green AR, Macmillan RD, Benhasouna A, Parr T, Ellis IO and Martin SG: Calpain system protein expression in basal-like and triple-negative invasive breast cancer. Ann Oncol 23: 2289-2296, 2012.

29. Storr SJ, Pu X, Davis J, Lobo D, Reece-Smith AM, Parsons SL, Madhusudan S and Martin SG: Expression of the calpain system is associated with poor clinical outcome in gastro-oesophageal adenocarcinomas. J Gastroenterol 48: 1213-1221, 2013.

30. Storr SJ, Zaitoun AM, Arora A, Durrant LG, Lobo DN, Madhusudan S and Martin SG: Calpain system protein expression in carcinomas of the pancreas, bile duct and ampulla. BMC Cancer 12: 511, 2012.

31. Baudry M, Chou MM and Bi X: Targeting calpain in synaptic plasticity. Expert Opin Ther Targets 17: 579-592, 2013.

32. Sawhney RS, Cookson MM, Omar Y, Hauser J and Brattain MG: Integrin alpha2-mediated ERK and calpain activation play a critical role in cell adhesion and motility via focal adhesion kinase signaling: Identification of a novel signaling pathway. J Biol Chem 281: 8497-8510, 2006.
33. Storr SJ, Thompson N, Pu X, Zhang Y and Martin SG: Calpain in breast cancer: Role in disease progression and treatment response. Pathobiology 82: 133-141, 2015.

34. Moretti D, Del Bello B, Allavena G and Maellaro E: Calpains and cancer: Friends or enemies? Arch Biochem Biophys 564: 26-36, 2014.

35. Pan Z, Chen C, Long H, Lei C, Tang G, Li L, Feng J and Chen F: Overexpression of GPC3 inhibits hepatocellular carcinoma cell proliferation and invasion through induction of apoptosis. Mol Med Rep 7: 969-974, 2013.

36. Song HH, Shi W, Xiang YY and Filmus J: The loss of glypican-3 induces alterations in Wnt signaling. J Biol Chem 280: 2116-2125, 2005.

37. Field MA, Cho V, Andrews TD and Goodnow CC: Reliably detecting clinically important variants requires both combined variant calls and optimized filtering strategies. PLoS One 10: $\mathrm{e} 0143199,2015$.

38. Earl J, Garcia-Nieto S, Martinez-Avila JC, Montans J, Sanjuanbenito A, Rodríguez-Garrote M, Lisa E, Mendía E, Lobo E, Malats N, et al: Circulating tumor cells (Ctc) and kras mutant circulating free Dna (cfdna) detection in peripheral blood as biomarkers in patients diagnosed with exocrine pancreatic cancer. BMC Cancer 15: 797, 2015. 